\title{
Conservation of adaptive potential and functional diversity
}

\author{
A. Rus Hoelzel ${ }^{1} \cdot$ Michael W. Bruford $^{2} \cdot$ Robert C. Fleischer $^{3}$
}

Published online: 8 February 2019

(c) Springer Nature B.V. 2019

\begin{abstract}
The conservation of adaptive potential to enable populations and species to respond to environmental change is one of the cornerstones of conservation genetics. To date, however, most work has by necessity focused on neutral markers and demographic questions. Now, with the rapid development of genomic technologies, we have new tools with which to address this essential but poorly understood aspect of conservation strategy. This was the motivation for a meeting on this subject held at Durham University in November 2017 that generated papers for this special issue. In this brief introduction we summarise the presentations and discussions that took place over the course of the meeting, and provide some initial conclusions together with ideas about the way forward.
\end{abstract}

\section{Introduction}

Conservation biology is a multi-faceted discipline dependent on the integration of biological, social and economic factors. The main objective of the growing sub-discipline of conservation genetics is the effective long-term conservation and management of biodiversity, necessary to protect ecosystems and their services. A tradition of investigating neutral genetic diversity to address questions about demography (population size, recruitment, inbreeding and connectivity) has served the purpose of more effective conservation well, and continues to be important, facilitating the identification of units for conservation. Neutral markers have also made an important contribution to forensics applications. However, phenotype is determined in part through adaptation at functional genes, and loci under positive selection can't be included in analyses based on neutral models (on which inference about demographic processes such as gene flow and population dynamics is based) without violating core assumptions. At the same time, it is natural selection at these

A. Rus Hoelzel

a.r.hoelzel@durham.ac.uk

1 Department of Biosciences, Durham University, South Road, Durham DH1 3LE, UK

2 School of Biosciences, University of Cardiff, Museum Avenue, Cardiff CF10 3AX, UK

3 Center for Conservation Genomics, Smithsonian Conservation Biology Institute, 3001 Connecticut Avenue, NW, Washington, DC 20008, USA functional loci that together with gene-environment interactions and phenotypic plasticity (including epigenetic effects) allow a population to respond to changing environments. Therefore, to preserve the potential for future adaptation, we need to ensure that we identify, characterise and conserve 'functional' diversity.

Methods integrating quantitative genetics and genomics are leading the way towards identifying relevant genes and pathways in non-model species. Inference from neutral loci linked to functional genes, or using neutral markers to estimate genome-wide processes, such as inbreeding and associations with fitness have also proved fruitful. However, these associations are typically weak, and populations differentiated at functional loci can be at the same time porous to neutral diversity, showing little or no differentiation at neutral markers. This means that conservation units defined using neutral genetic markers can hide cryptic patterns of differentiation, the conservation of which could be crucial to protect the long-term viability of a species. Some studies focusing on specific genes have illustrated this, such as the association between immune system gene diversity and rabies exposure in racoon (Procyon lotor) populations (Kyle et al. 2014). Addressing the question more widely is at last becoming possible due to an improved understanding of the relationship between genotype and phenotype (see Kratochwil and Meyer 2015), better methods to model and detect selection (e.g. Nielsen et al. 2010), and our ability to screen whole genomes at the population level (see Soria-Carrasco et al. 2014, who screened eight populations to assess divergence among stick insects adapted to different hosts). 
Harrison et al. (2014) discuss the use of genomics to characterise evolutionary potential and to predict the efficacy of evolutionary rescue (rapid adaptation to escape extinction). Even forensic application can be facilitated, such as the use of gene-associated markers to help regulate illegal fisheries (e.g. Nielsen et al. 2012). At the same time, there remain important challenges. For example, Poh et al. (2014) illustrate difficulties with detecting the signal for selection in populations that have been through a severe bottleneck, and progress with understanding genotype-phenotype relationships have so far mostly been with model organisms. Funk et al. (2012) and others have emphasized the importance of developing a new paradigm that incorporates functional loci when defining conservation units. This reflects a key focus on the identification of cryptic diversity associated with local adaptation. Gene-targeted approaches based on single loci of conservation concern either in captive or heavily managed wild populations may also be possible, and there are ongoing programs attempting this, but there are various challenges and risks especially related to the concomitant impact on the rest of the genome (see Kardos and Shafer 2018).

Identifying strategies to conserve adaptive potential is a challenging but critical problem. Anthropogenic environmental change intensifies the risks associated with this challenge, because affected populations must move, adapt, or go extinct. Biodiversity conservation, which therefore must include functional diversity, supports societal needs in a diversity of ways. Biodiversity is a core component of ecosystem resources in support of economic sustenance and future development. It is also at the heart of drug discovery and the development of new medicines. The core objective for this meeting was therefore translational by definitionabout the effective, long-term conservation of biodiversity. However, it was also about understanding essential processes in evolutionary biology. For years conservation genetics has concentrated on understanding neutral evolutionary processes and the implications for species survival. Now we need to incorporate a better understanding of the role of natural selection, not that it wasn't important before, but the tools to more thoroughly address the relevant questions are now available. There is still some ways to go, but it is now possible to identify the best way forward.

\section{The meeting and discussions}

The meeting was held over 2 days and included 24 oral presentations and sessions for open discussion. The main themes were on ways to identify loci of interest, the interaction between selection and drift in small populations, selective responses to pathogens, and ways to integrate an understanding of adaptive processes into more effective conservation and management policy. Presentations considered the influence of environmental gradients on adaptive diversity, such as differentiation along an altitudinal gradient in the saker falcon (see Pan et al. 2017) or across the mesopelagic, bathypelagic transition in the deep sea for a demersal fish species (see Gaither et al. 2018). Bumblebee genomics (see Sadd et al. 2015) were discussed for their potential to reveal adaptive changes in the context of land use histories. These methods are best at identifying a few loci of apparently major effect, though how much phenotypic diversity is instead determined by many loci of small effect remains a focus of research (e.g. Pieffer et al. 2014). Another example at the meeting discussed the few genes that determine wing colouration patterns in Heliconius butterflies, and the importance of introgression between populations in generating those patterns (see Martin and Jiggins 2017). Hybridisation between wolves and domestic dogs was discussed as another possible route to adaptive variation gained through introgression (see Pilot et al. 2018). Presentations about deep sea demersal fish and shark species illustrated differential patterns of population structure (based on RADseq analyses) when outlier loci (putatively under selection) are compared to patterns based on neutral loci, and posed the question as to whether this may be sufficient information to identify populations of conservation concern, even without the identification of specific relevant loci and their functions. The phylogenetic approach to identifying loci under selection was represented by a study on kiwi evolution, where the comparison of transcriptomes revealed signals for positive selection in 77 orthologs on the branch separating kiwi species from other taxa (see Ramstad et al. 2016).

In the special issue we focus on four studies that cover a breadth of topics under the theme of identifying loci under selection. Munoz-Fuentes et al. (2018) report on the international mouse phenotyping consortium (IMPC) database as a resource for identifying candidate loci potentially under selection in mammalian species. This database derives information from experimental evidence using knockout lines for all protein coding loci, something that is of course unavailable for wildlife species of conservation concern. In the mouse $\sim 35 \%$ of screened loci are apparently essential for viability, and the authors propose that this could be the case for a wide range of other mammalian species. Some initial analyses identify some of these 'loss of function' (LoF) loci in the gorilla and other wildlife species. Bucharova et al. (2019) also suggest an indirect approach to promote the conservation of adaptive potential, in this case for ecosystem restoration projects re-establishing plant populations. They suggest a program of 'regional admixture provenancing', mixing seeds to promote local diversity, but only on a regional scale to avoid outbreeding depression, and review experimental data that supports this strategy. Hauffe and Barelli (this issue) consider the promotion of adaptive 
potential in the context of an organism's microbiota. Just as gene-environment interactions are important with respect to external interactions, commensal microbiota composition and function are likely to shape adaptive potential, and this review considers the few data currently available. Finally, Hendricks et al. (this issue) review the population genomics of the gray wolf, which provides a data-rich example of inference from genomic data towards the identification of genes associated with the 'adaptive uniqueness' of regional wolf populations.

For many populations or species of conservation concern there are low or diminishing numbers, and in small populations genetic drift is expected to be a stronger force than selection. At the meeting there were two presentations (by students Menno de Jong and Deborah Leigh) about the difficulty in identifying signals for selection when population size is small, due to the confounding signal from genetic drift. In one case the authors sought to reveal loci under selection by comparing parallel founder events, and in both presentations they compared results against expectations from simulations. Although some relevant loci could be discovered, others likely remain obscured by stochastic noise. Another presentation pointed out the value of considering data from livestock populations, where artificial selection leads to strong inbreeding and the loss of diversity, but the retention of adaptive potential is a key objective (see Eynard 2018). A presentation on the genome-wide changes seen in the last surviving woolly mammoth population at neutral and functional loci emphasized the dominance of drift over selection in small populations (c.f. Pečnerová et al. 2017).

In the special issue we focus on three studies that consider adaptive potential in the context of demography. Following a presentation that reviewed the population genomic data on stick insect (Timema sp.) population connectivity and local adaptations, Nosil et al. (this issue) present a simulation analysis on how local and system wide adaptation is influenced by population connectivity. With carrying capacity per deme set at 100 individuals, they show that increased connectivity among sub-populations leads to 'sudden and system-wide adaptation' over-riding changes at a more local scale. When natural connectivity is not an option, the introduction of individuals into small and endangered populations is sometimes considered to promote 'genetic rescue' (see Whiteley et al. 2015). However, there are consequences associated with this process. Harris et al. (this issue) using simulations and a natural example comparing Neanderthals and humans show how genetic rescue can lead to the partial loss of native genome content. They find that the increase in fitness associated with genetic rescue is accompanied by an approximately proportional reduction in the fraction of native genome preserved, and that the duration and nature of a bottleneck affects the strength of fitness benefits. The final paper on this theme revisits a well-known example of a natural population that is genetically depauperate, the cheetahs (Acinonyx jubatus) of Namibia (Schwensow et al. this issue). Based on sequence and expression data for MHC class I and class II loci, this study confirms low immune system genetic diversity for this species, but the authors suggest that immune function was based on more complex interactions than reflected in sequence diversity alone.

The importance of preserving MHC diversity to conserve the ability of a population to respond to novel pathogens has been recognised and discussed in the literature since the 1980s. At the meeting a study based on 1675 guppies was presented, addressing the apparent paradox between the reduction of diversity generated from an 'arms race' driven by host-parasite dynamics and the long-term persistence of MHC diversity by 'trans-species' evolution. The authors found that subdivision of MHC function into 'supertypes' maintained diversity as a balanced polymorphism, inference supported by both empirical data and simulations (see Lighton et al. 2017). Another presentation indicated that selfing species may present differential responses to the pathogen environment by adjusting patterns of gene expression. These examples reflect the complexities of the evolutionary process, but it remains clear that conserving relevant immune system diversity is an essential aspect of effective conservation, especially in an age when the transfer of exotic pathogens is a problem of increasing frequency. A presentation reviewed this issue with a focus on fungal infection in amphibians and avian malaria in Hawaiian honeycreepers. In the case of the amphibian study, RNAseq data after experimental infection revealed the role of MHC super-types in eliciting a massive immune response in dying versus surviving leopard frogs, and directional selection in response to fungal infection was implicated in the reduction of MHC diversity in a related species (see Savage et al. 2016, 2017). For honeycreepers, genomic analyses identified candidate loci that may facilitate survival after malarial infection in the amakihi (see Cassin-Sackett et al. 2018). In the special issue we include one example based on the genomic context of susceptibility of the Tasmanian devil to facial tumours (Hohenlohe et al. this issue). This study reviews recent findings from genome scans of loci associated with this disease, and proposes strategies for the more effective management of infection to avoid the previously predicted outcome of extinction.

The final theme was on how the conservation of adaptive potential can be better integrated into conservation and management policies. At the meeting there was a presentation on the importance of a multi-taxon approach to conserving 'environmentally associated variation', noting that although neutral markers may indicate the general trend, in the future we need to incorporate inference from functional loci more fully (see Thomassen et al. 2011). Another presentation reviewed the importance 
and potential for measuring both adaptive and deleterious variation in threatened populations (see Freedman et al. 2016). In the special issue Mable (2019) acknowledges the importance of genomic methods towards the conservation of adaptive potential, but suggests that integration with ecological methods and concepts remains essential. She stresses the importance of work towards understanding adaptive processes, beyond merely descriptive data. Stanton et al. (this issue) frame the question in the context of species delimitation, advocating a genomic approach and arguing that an appropriate species concept (neither over-splitting nor inappropriate merging) is an important aspect of the effective conservation of functional (phenotypic) diversity and adaptive potential. Finally Funk et al. (this issue) note that rapid environmental change makes the conservation of adaptive potential more important than ever. They describe how this may be achieved under the framework of the US endangered species act (ESA) and by inference for other legislative mechanisms governing environmental policy elsewhere in the world. While recognising that the complexities of adaptive systems may prevent a full understanding, they propose that the best available evidence regarding adaptive potential should be being incorporated into modelling and decision making processes now.

In discussion sessions the heterogeneity of legislative frameworks for biodiversity conservation across Europe was noted, and the poor representation of conservation genetics in formal processes. The advantages of the US system were considered, especially with respect to the common integration of genetic data, the formal process for the designation of 'distinct population segments' for conservation allowed under the US ESA (independent of issues associated with taxonomic classification), and the recording of the application and decision process in the Federal Register. At the same time it was noted that this can be a difficult process when financial considerations lead to litigation, and these cases can lead to calls to limit or weaken the ESA. An international working group was proposed to specifically promote the development of policy that would protect adaptive potential in natural populations, and the importance of engaging the public was emphasised. In terms of practical considerations, there was a suggestion to preserve variation that coalesces deeply, given that this may reflect adaptive differences retained over time. The best approach to managing hybrids was discussed in some detail. While some are keen on preserving pure lines, natural hybridisation can be a mechanism promoting diversity and adaptive potential. It was suggested that clearly disruptive processes leading to outbreeding depression or the loss of pure lines due to anthropogenic interference should be distinguished from natural introgression in support of conservation strategies for listing and managing hybrids.

\section{Conclusions}

While we are in the early stages of this important transition, new methodologies and the recognition that we need to use the large datasets now available to help understand evolutionary process are providing the mechanisms necessary to incorporate adaptive potential into our conservation strategies. Genomic methods provide high resolution for addressing demographic questions, which remain important, but these methods also facilitate the identification of evolved functional differences among populations that need to be conserved. In some cases the need is targeted, such as in association with resistance against pathogens, while in many cases it is just about conserving adaptive potential in a changing world. This meeting helped to identify the challenges still ahead, but also provided a wealth of examples of the progress already made.

Acknowledgements Thanks to those who helped with the organisation and running of the meeting, especially Menno de Jong and Thomas Keggin who helped out with registration and session chairing on the day, and the event office at Durham University who organised registration and catering. We also thank our sponsors, Springer-Nature and the Genetics Society.

\section{References}

Bucharova A, Bossdorf O, Holzel N, Kollmann J, Prasse R, Durka W (2019) Mix and match: regional admixture provenancing strikes a balance among different seed-sourcing strategies for ecological restoration. Con Genet. https://doi.org/10.1007/s1059 2-018-1067-6

Cassin-Sackett L, Callicrate TE, Fleischer RC (2018) Parallel evolution of gene classes, but not genes: evidence from Hawai'ian honeycreeper populations exposed to avian malaria. Mol Ecol. https:// doi.org/10.1111/mec.14891

Eynard SE (2018) Using genomic information to conserve genetic diversity in livestock. PhD Thesis, Wageningen University, Wageningen, The Netherlands

Freedman AH, Lohmueller KE, Wayne RK (2016) Evolutionary history, selective sweeps and deleterious variation in the dog. Ann Rev Ecol Evol Syst 47:73-96

Funk WC, McKay JK, Hohenlohe PA, Allendorf FW (2012) Harnessing genomics for delineating conservation units. TREE 27:489-496

Funk WC, Forester BR, Converse SJ, Darst C, Morey S (2019) Improving conservation policy with genomics: a guide to integrating adaptive potential into US endangered species act decisions for conservation practitioners and geneticists. Conserv Genet. https ://doi.org/10.1007/s10592-018-1096-1

Gaither MR et al (2018) Genomics of habitat choice and adaptive evolution in the deep sea. Nat Ecol Evol 2:680-687

Harris K, Zhang Y, Nielsen R (2019) Genetic rescue and the maintenance of native ancestry. Conserv Genet. https://doi.org/10.1007/ s10592-018-1132-1

Harrison A, Pavlova A, Telonis-Scott M, Sunnucks P (2014) Using genomics to characterize evolutionary potential for conservation of wild populations. Evol Appl 7:1008-1025 
Hauffe HC, Barelli C (2019) Conserve the germs: the gut microbiota and adaptive potential. Conserv Genet. https://doi.org/10.1007/ s10592-019-01150-y

Hendricks SA, Schweizer RM, Wayne RK (2019) Conservation genomics illuminates the adaptive uniqueness of North American gray wolves. Conserv Genet. https://doi.org/10.1007/s1059 2-018-1118-Z

Hohenlohe PA, McCallum HI, Jones ME, Hamede RK, Storfer A (2019) Conserving adaptive potential: lessons from Tasmanian devils and their transmissible cancer. Conserv Genet (this issue)

Kardos M, Shafer ABA (2018) The peril of gene-targeted conservation. TREE 33:827-839

Kratochwil CF, Meyer A (2015) Closing the genotype-phenotype gap: emerging technologies for evolutionary genetics in ecological model vertebrate systems. BioEssays 37:213-226

Kyle CJ, Rico Y, Castillo S, Srithayakumar V, Cullingham CI, White BN, Pond BA (2014) Spatial patterns of neutral and functional genetic variations reveal patterns of local adaptation in raccoon (Procyon lotor) populations exposed to raccoon rabies. Mol Ecol 23:2287-2298

Lighten J, Papadopulos AST, Mohammed PS, Ward BJ, Paterson IG, Baillie L, Bradbury IR, Hendry AP, Bentzen P, van Oosterhout C (2017) Evolutionary genetics of immunological supertypes reveals two faces of the Red Queen. Nat Comm 18:1294

Mable BK (2019) Conservation of adaptive potential and functional diversity: integrating old and new approaches. Conserv Genet. https://doi.org/10.1007/s10592-018-1129-9

Martin SH, Jiggins CD (2017) Interpreting the genomic landscape of introgression. Curr Opin Genet Dev 47:69-74

Muñoz-Fuentes V et al (2018) The International Mouse Phenotyping Consortium (IMPC): a functional catalogue of the mammalian genome that informs conservation. Conserv Genet 19:995-1005

Nielsen R, Hubisz MJ, Hellmann I, Torgerson D, Andres AM, Albrechtsen A, Gutenkunst R, Adams MD, Cargill M, Boyko A, Indap A, Bustamante CD, Clark AG (2010) Darwinian and demographic forces affecting human protein coding genes. Genome Res 19:838-849

Nielsen EE et al (2012) Gene-associated markers provide tools for tracking illegal fishing and false eco-certification. Nat Comm 3:851

Nosil P, Soria-Carrasco V, Feder JL, Flaxman SM, Gompert Z (2019) Local and system-wide adaptation is influenced by population connectivity. Conserv Genet. https://doi.org/10.1007/s1059 2-018-1097-0

Pan S et al (2017) Population transcriptomes reveal synergistic responses of DNA polymorphism and RNA expression to extreme environments on the Qinghai-Tibetan Plateau in a predatory bird. Mol Ecol 26:2993-3010

Pečnerová P, Palkopoulou E, Wheat $\mathrm{CW}$, Skoglund P, Vartanyan S, Tikhonov A, Nikolskiy P, van der Plicht J, Diez del Molino D,
Dalen L (2017) Mitogenome evolution in the last surviving woolly mammoth population reveals neutral and functional consequences of small population size. Evol Lett 1:292-303

Peiffer JA, Romay MC, Gore MA, Flint-Garcia SA, Zhang Z, Millard MJ, Garndner CAC, McMullen MD, Holland JB, Bradbury PJ, Buckler ES (2014) The genetic architecture of maize height. Genetics 196:1337-1356

Pilot M, Greco C, vonHoldt BM, Randi E, Jedrzejewski W, Sidorovich VE, Konopinski MK, Ostrander EA, Wayne RK (2018) Widespread, long-term admixture between grey wolves and domestic dogs across Eurasia and its implications for the conservation status of hybrids. Evol Appl 11:662-680

Poh Y-P, Domingues VS, Hoekstra HE, Jensen JD (2014) On the prospect of identifying adaptive loci in recently bottlenecked populations. PLoS ONE 9:e110579

Ramstad KM, Miller HC, Kolle G (2016) Sixteen kiwi (Apteryx spp) transcriptomes provide a wealth of genetic markers and insight into sex chromosome evolution in birds. BMC Genom 17:410

Sadd BM et al (2015) The genomes of two key bumblebee species with primitive eusocial organisation. Genome Biol 16:76

Savage AE, Terrell KA, Gratwicke B, Mattheus NM, Augustine L, Fleischer RC (2016) Reduced immune function predicts disease susceptibility in frogs infected with a deadly fungal pathogen. Conserv Physiol 4:cow011

Savage AE, Mulder KP, Torres T, Wells S (2017) Lost but not forgotten: MHC genotypes predict overwinter survival despite depauperate MHC diversity in a declining frog. Conserv Genet 19:309-322

Schwensow N, Castro-Prieto A, Wachter B, Sommer S (2019) Immunological MHC supertypes and allelic expression: how low is the functional MHC diversity in free-ranging Namibian cheetahs? Conserv Genet. https://doi.org/10.1007/s10592-019-01143-x

Soria-Carrasco V et al (2014) Stick insect genomes reveal natural selection's role in parallel speciation. Science 344:738-742

Stanton DWG, Frandsen P, Waples RK, Heller R, Russo IM, Orozco PA, Tingskov-Pedersen C-E, Siegismund HR, Bruford MW (2019) More grist for the mill? Species delimitation in the genomic era and its implications for conservation. Conserv Genet. https://doi. org/10.1007/s10592-019-01149-5

Thomassen HA et al (2011) Mapping evolutionary process: a multitaxa approach to conservation prioritization. Evol Appl 4:397-413

Whiteley AR, Fitzpatrick SW, Funk WC, Tallmon DA (2015) Genetic rescue to the rescue. TREE 30:42-49

Publisher's Note Springer Nature remains neutral with regard to jurisdictional claims in published maps and institutional affiliations. 